\title{
Tensiones en el circuito cinematográfico internacional: modelo para el estudio de los festivales latinoamericanos
}

Inequalities within the international film arena. A framework to studying Latin American film festivals

\section{Minerva Campos-Rabadán}

Universidad Autónoma de Madrid, Madrid, España

minervacamposrabadanagmail.com

https://orcid.org/0000-0001-6984-500X

\section{Resumen}

Las tensiones y diálogos asimétricos entre centros y periferias que se evidencian en los estudios dedicados a festivales de cine y a fenómenos que tienen lugar en dicho contexto, están estrechamente relacionados con la importancia de los eventos que conforman el circuito internacional. Este artículo desarrolla un modelo para el análisis de dichas relaciones a partir del circuito latinoamericano y de un marco teórico que toma elementos del propio campo de los Film Festival Studies y de las Teorías del Sistema Mundo desarrolladas en los años setenta.

Palabras clave: festivales de cine, cine latinoamericano, cine periférico, centro-periferia, propuesta metodológica.

\begin{abstract}
The unequal dialogues and tensions between centres and peripheries that rule the international film context referred to by Film Festival studies. The idea is directly related with the differences in importance that experts note among each one of the festivals that constitute the international circuit. The main purpose of this article is to set a model for the analysis of these unequal relationships among events. It will consider the Latin American film festival circuit and key concepts provided by the Film Festival Studies and the World-System Theories developed during the 1970s.
\end{abstract}

Keywords: film festivals, Latin American cinema, peripheral cinema, core-periphery, centre-periphery, methodological proposal. 


\section{Introducción}

En tres décadas de investigaciones adscritas a los Film Festival Studies, la atención a nuevos fenómenos, eventos, dinámicas, agentes y profesionales, creadores, estéticas, secciones, geografías y subcircuitos ha dado cuenta de la complejidad y diversidad propias de este campo. Sin embargo, y a pesar de la vasta bibliografía especializada, el desarrollo de marcos teóricos y metodologías para el estudio de estas cuestiones ha sido más limitado. Entre los aportes más reseñables se cuentan el de Marijke de Valck en Film Festivals: From European Geopolitics to Global Cinephilia (2007), cuya propuesta se articula a partir de la teoría del Actor-Red de Bruno Latour (2008). Siguiendo el esquema de Latour, de Valck establece un modelo para el estudio integral de los festivales que considera todos los elementos presentes en dichos eventos (desde las películas hasta los visitantes y los profesionales de la crítical. Tanto el trabajo de De Valck como los marcos teóricos en los que se apoya son referencia fundamental en este campo. Destacan también propuestas desarrolladas desde la antropología y los estudios de las artes y la cultura. En esta línea, el congreso internacional celebrado en París en 2011 y su publicación derivada, Une histoire des festivals (2013), constituyen otra de las claves en el campo, dado que sitúan decididamente los festivales de cine en la tradición de los festivales culturales, ubicándolos así en un contexto disciplinar más amplio (Fléchet et al., 2013)'. Por su orientación antropológica, también destaca Film Festivals and Anthropology (Vallejo \& Peirano, 2017), una publicación colectiva que aborda las complejas dinámicas que se despliegan en estos eventos, proponiendo, al mismo tiempo, metodologías específicas para estudiar el circuito y los fenómenos que le son propios.

Con el objetivo de contribuir al estudio de las sinergias y tensiones que tienen lugar en este contexto, este artículo desarrolla una propuesta teóricometodológica que permita analizar la importancia, la influencia y el poder que cada evento particular tiene dentro del circuito internacional y en los diferentes subcircuitos delimitados para su estudio. Aunque los festivales actúen como mediadores entre los cines del mundo (en tanto que lugares de encuentro, diálogo y configuración de categorías), consideramos que no eliminan ni reducen las asimetrías que se verifican en el contexto cinemato- gráfico internacional y que aquí analizaremos en términos de centros y periferias. Entendemos que mantienen y alimentan dichas tensiones porque los festivales en sí mismos se relacionan entre ellos sobre la base de un esquema jerárquico que, también, analizamos bajo la óptica centros/periferias. Es precisamente esta hipótesis la que guía nuestro trabajo.

Para analizar la importancia y el papel de los festivales celebrados en América Latina en el circuito internacional y en subcircuitos más específicos, discutiremos, primero, las desigualdades que surgen al enfrentar esta región cinematográfica considerada periférica a otra debido a la cual detenta, precisamente, este estatuto y que, por oposición, resultaría central en el contexto cinematográfico contemporáneo. El análisis se centra, inicialmente, en las tensiones que emergen en el circuito internacional, para dirigir, luego, la atención al poder, la influencia y la importancia que en este y otros marcos tendrían los festivales de cine celebrados en América Latina.

\section{Marco teórico}

Si atendemos al cine en su dimensión global Estados Unidos y Europa emergen como dos ejes centrales que articulan y en torno a los cuales se definen los cines de todo el mundo. En el primer caso, la importancia de Estados Unidos sería una consecuencia directa de su hegemónica industria cinematográfica. En el segundo, la importancia de Europa se relaciona, más bien, con el poder de legitimación de sus instituciones cinematográficas (Crofts, 1993; Elena, 1999; Czach, 2004). La articulación de estas tensiones en un esquema de centros y periferias, en unos términos que desarrollaremos más adelante, supone que el cine está organizado en torno al eje euro-norteamericano y "el resto del mundo". Se trata de una polarización presente también en otras categorías como las de Tercer Cine, Cine del Tercer Mundo, World Cinema o Cine del Sur, a veces utilizadas como equivalentes entre ellas y como sinónimos de cine periférico (Campos, 2016b).

En todos los casos, estos conceptos se refieren a cines (trans)nacionales menos comerciales, menos industrializados y menos influyentes que, además, 
reciben menos atención internacional ly, en términos gruesos, se producen en África, Asia y América Latina). Si bien desde una perspectiva teórica parece superada la cuestión nacional que está en la base de estas categorías, en el ámbito cinematográfico sigue siendo un criterio operativo tanto para los festivales que aquí nos ocupan, como para la academia y la industria. En el caso de los primeros, supone uno de los criterios tradicionales para la selección, organización y descripción de las películas que integran cada una de sus secciones, aunque los procedimientos hayan variado a lo largo del tiempo y en cada festival (Campos, 2018). También en el ámbito de la producción y la distribución cinematográficas, la categoría de lo nacional continúa regulando la participación y los acuerdos de empresas ubicadas en diferentes territorios, lo que, a su vez, afecta a las políticas de fomento de distinto alcance (estatales, regionales, internacionales) y a las estrategias de distribución internacional.

Como en el caso de lo nacional, la ecuación centroperiferia es objeto de un vivo debate sobre su obsolescencia (Appadurai, 1996, p.46) al tiempo que sigue siendo operativa en el ámbito cinematográfico. Se trata de dos conceptos opuestos que son citados con frecuencia (en el contexto profesional y académicol y que parecen haber cristalizado como categorías no problemáticas, obviando las dinámicas y mecanismos que las han configurado y que, a lo largo del tiempo, han reproducido y consolidado relaciones desiguales entre las cinematografías del mundo (sus instituciones, sus agentes, sus obras y sus políticas). El propósito de este trabajo es, por ello, visibilizar las dinámicas y tensiones implícitas cada vez que nos referimos a cinematografías y festivales en estos términos.

Revisamos algunos conceptos de los estudios sobre festivales de cine que resultan clave para comprender un contexto cinematográfico dividido en dos grandes áreas tensamente conectadas por relaciones de poder desiguales. Con el objetivo de identificar las características principales de las regiones consideradas centrales y periféricas, atenderemos a las conceptualizaciones teóricas propuestas por Johan Galtung (1971) e Immanuel Wallerstein $(1974,1976)$ en los campos de la economía y la política. Analizaremos también el modo en que cada festival opera según este modelo de centros y periferias y cómo la tensión entre ellos toma forma en el circuito internacional de festivales de cine y en subcircuitos más acotados. Por últi- mo, revisaremos también la hipótesis de los guetos de programación y exploraremos la importancia de las jerarquías descritas al momento de configurar y legitimar categorías cinematográficas, de nuevo, tomando los festivales de América Latina como punto de apoyo.

\section{Conceptos clave de los Film Festival Studies: festivales, jerarquías y (sub)circuitos}

Esta sección discute los conceptos de los Film Festival Studies que se refieren al objeto "festival de cine" y al modo en que estos eventos se relacionan entre ellos. Cada aproximación al festival de cine ha dado como resultado una definición distinta. Janet Harbord (2002), por ejemplo, considera los festivales de cine como parte integrante de un circuito de distribución inicial ${ }^{2}$ y los describe como un espacio situado entre los intereses económicos, el conocimiento especializado y el turismo (pp. 2-67).

En nuestra propuesta subrayamos elementos propios del festival de cine que permiten, a partir de su estudio independiente, múltiples aproximaciones al campo y que, por otro lado, resultan también útiles a la hora de organizar y delimitar subcircuitos específicos en base a las características que comparten. Los festivales de cine consisten en la puesta a disposición de una serie acotada de contenidos audiovisuales durante un periodo de tiempo determinado en un espacio definido (aunque no único). Esta dinámica generaría un marco de encuentro para diferentes públicos (generales y/o profesionales) interesados en las películas incluidas en el programa (películas que podrían presentar diferentes géneros, duraciones y formatos). La puesta a disposición tiene que ver con que los títulos propuestos por un festival en cada una de sus ediciones ya no solo se corresponden con proyecciones, sino que, muchas veces, los eventos deslocalizan una parte de su programación al espacio de internet o son, en otros casos, festivales concebidos para ser celebrados online. Es por ello que dejamos de lado la idea de un lugar físico y un espacio único, considerando tanto la multiplicidad de pantallas como los casos de festivales itinerantes (como Ambulante. Cine Documental Itinerante en Méxicol o los que de manera simultánea se celebran en diferentes lugares del mundo (como Márgenes, que en su edición 
de 2017 contó con proyecciones en Madrid, Córdoba, Barcelona, Ciudad de México, Santiago de Chile y Montevideo, además de haber programado títulos disponibles online).

Otro elemento que caracteriza al festival es que al menos algunas de las películas seleccionadas forman parte de una sección competitiva y son, por ello, candidatas a alguno de los premios convocados en cada edición. Existe una animada discusión acerca de si son las secciones competitivas uno de los criterios que permiten diferenciar un festival de cine de una muestra u otro tipo de encuentro. En 2020, el Festival Internacional de Cine de Cartagena de Indias, por ejemplo, se concibió por primera vez como un festival sin premios, lo que dio lugar a un amplio debate en el ámbito de la programación, la crítica y la academia sobre lo adecuado de esta decisión, así como la de reformular la idea misma de festival para incluir eventos que prescinden de todas sus secciones competitivas. En principio, y teniendo en cuenta tanto la dimensión económica de los premios (que habitualmente implican una cantidad en metálicol como el interés mediático que despiertan (durante el tiempo en que se exhiben las películas a competición, en el momento de la premiación y en las crónicas resumen del festival), en este trabajo consideramos las secciones competitivas uno de los elementos definitorios de los festivales de cine, aunque en función del estudio propuesto y la perspectiva adoptada haya excepciones que redefinen éste u otros parámetros de análisis.

Otra idea clave es la de circuito. El circuito internacional de festivales surgió en el momento mismo en el que las referencias de los nuevos certámenes eran, precisamente, los festivales ya existentes y configuraban sinergias y competencias mutuas, como sigue ocurriendo. Proponemos una definición amplia de circuito de festivales susceptible de aplicarse a otros espacios cinematográficos. Un circuito consiste en un grupo de eventos (o pantallas) que comparten todas o algunas de las características antes señaladas y que constituyen un itinerario posible para películas. Además, todos los festivales pertenecientes al circuito internacional estarían conectados en la medida en que están informados y prestan atención a las operaciones, selecciones y premios que tienen lugar en el resto de los eventos que integran el grupo o circuito. Es posible también determinar subcircuitos más acotados dependiendo de las características compartidas por los festivales individuales incluidos en cada uno. Los subcircui- tos son más restringidos y articulados en torno a criterios diversos como su línea de programación (un subcircuito dedicado al cine latinoamericano), su celebración en un lugar concreto (festivales celebrados en América Latina), en determinado tipo de pantallas (proyecciones en salas de cine o exhibición en streaming), en función de su formato (subcircuitos de súper-8 o cine digital), su duración (de corto, largo o medio metraje) o su género (de cine trash o político). Existen diferentes criterios para delimitar subcircuitos independientes con calendarios propios que, dedicados a diferentes películas, existen de manera simultánea y en paralelo al circuito internacional (lordanova, 2009).

La tercera idea clave es la de jerarquía. Diversos autores se han ocupado de las jerarquías que organizan el circuito internacional y el modo en que cada festival detenta una posición y un papel determinado en ella (De Valck, 2007; Iordanova, 2009). En el caso del circuito internacional, Julian Stringer (2001) destaca la desigualdad que reina entre los festivales que lo integran y señala el binomio centro-periferia que define sus relaciones: "los eventos se miden y comparan, se diferencian perfiles altos de bajos, lugares glamorosos y sexies separados de los no tan glamurosos ni tan sexies. La desigualdad se construye entonces dentro de la estructura del circuito internacional de festivales de cine", siendo este circuito parte de las nuevas relaciones "centro-periferia" que tienen lugar entre diferentes naciones y regiones (p.138).

De Valck (2014) aborda las jerarquías de los festivales estableciendo tres categorías distintas que podemos definir como de negocio y articula tres niveles sobre la base de la importancia de los mercados y el prestigio de las competiciones de los casos que analiza (p.47). Aunque la autora deja de lado en ese artículo las dinámicas geopolíticas que afectan esta clasificación, otros especialistas han subrayado las relaciones de poder desiguales que operan entre festivales situados en regiones distintas (Stringer, 2016, p.38). Al analizar las colaboraciones que se dan entre festivales de mayor y menor tamaño se identifican relaciones de naturaleza jerárquica (Falicov, 2017).

Siguiendo las propuestas de Stringer y de Valck, existen eventos de primera y segunda clase. Sin embargo, es necesario poner el foco más allá: en la importancia relativa y diferenciada que tiene cada festival si consideramos su papel en el circuito internacional y en alguno de los subcircuitos que 
integra. Estas dinámicas de naturaleza geopolítica también operan en los subcircuitos temáticos y especializados.

\section{Los conceptos de centro y periferia}

El esquema centro-periferia (en la medida en que se refiere a territorios geopolíticos) se relaciona con conceptos como el de lo nacional y lo regional. Aunque el binomio centro-periferia y las categorías de lo nacional y lo regional parecen obsoletas en un mundo contemporáneo regido por acuerdos transnacionales y mercados globales o globalizados, son términos altamente operativos porque consideran la coexistencia de dos fenómenos simultáneos que afectan al cine de todo el mundo. Por un lado, existe una complejidad creciente en las narrativas híbridas transnacionales, en los mercados globales y los acuerdos de producción y distribución transnacionales. Por otro lado, se produce una "nacionalización" de películas en el sentido en que con frecuencia son asociadas a uno o varios países y a regiones concretas de todos cuantos aparecen en sus créditos de producción, un fenómeno que tiene especial incidencia en el caso de territorios pertenecientes a regiones periféricas. Algunos autores han señalado la asociación entre películas y países o regiones en términos comerciales y promocionales (Ross, 2011; Crofts, 1999). Estos fenómenos globalizan mercados al mismo tiempo que eliminan fronteras, mientras que el otro establece categorías y etiquetas basadas en demarcaciones territoriales (nacionales o regionales). En lo que nos atañe, ambas operaciones cuentan con los festivales de cine como espacio privilegiado y son una constante en su historia (Campos, 2018).

Johan Galtung (1971) e Immanuel Wallerstein (1974, 1976) proponen esta categorización dual del mundo en centros y periferias. Las relaciones desiguales entre territorios han sido previamente exploradas por los estudios coloniales, postcoloniales y las teorías de la dependencia. Considerando estos antecedentes, destacamos la idea de desarrollo y subdesarrollo como dos caras de una misma moneda y de comportamientos derivados de las relaciones coloniales en las cuales el centro ejerce influencia sobre la periferia ${ }^{3}$; nos referimos al poder que los festivales del centro ejercen sobre el resto de los eventos que conforman el circuito internacional.
Galtung (1971) describe el mundo como un sistema polarizado regido por la desigualdad y organizado en áreas centrales y periféricas como resultado de estas relaciones. De acuerdo con esto, el mundo está dividido en centros y periferias y cada una de estas dos divisiones tiene sus propios centros y periferias ${ }^{4}$. En su análisis, Galtung identifica diferentes modelos relacionales entre las cuatro áreas descritas. Uno de ellos es un modelo positivo y colaborativo que resulta en un esquema de transacciones e influencias que pueden ser resumidas como de los centros a las periferias ( $C-P$; $C-p ; c-p)$, entre periferias ( $P-p)$ y entre centros (C-c) (p.106). Este modelo es especialmente apropiado para el estudio de los festivales de cine porque consideramos que los eventos se relacionan en este circuito de una manera colaborativa lindependientemente de la competencia que tiene lugar entre los festivales por conseguir películas, patrocinadores, oportunidades de negocio o por las propias fechas en el calendariol. Además, el modelo de Galtung señala que algunas áreas son relativamente estables en el centro y en la periferia, en parte como resultado de los procesos que tienen lugar en ambas áreas y que consolidan y refuerzan la situación de cada una. El autor señala cómo, "al aceptar la transmisión cultural, la Periferia también, implícitamente, valida para el Centro la cultura desarrollada en el centro, sea ese centro intra- o internacional. Esto refuerza al Centro como centro [...]" (p. 93). Es decir, los festivales localizados en la periferia del circuito contribuyen a mantener la posición privilegiada de los eventos centrales.

En su investigación, Galtung también señala la importancia de estudiar estos fenómenos en contextos geopolíticos concretos analizando la "interacción entre naciones o grupos de naciones" (p.85). Es posible analizar cómo los festivales periféricos siguen e imitan los modelos y mecanismos (secciones, actividades, fórmulas, espacios de negocio y de formación) puestos en marcha inicialmente por festivales centrales. Además, los festivales periféricos están interesados en las tendencias cinematográficas visibilizadas en mayor medida Ino necesariamente "descubiertas" o previamente exhibidas) por eventos situados en el centro del circuito internacional y de los subcircuitos de los que forman parte, replicando ciertas tendencias y consolidando así la influencia que los eventos centrales ejercen sobre el resto del (sub)circuito. 
Dado el gran número de festivales que se celebran en todo el mundo, es necesario subrayar la posibilidad de que coexistan varios centros simultáneos. Hay festivales con un poder e influencia muy similares y que, en este sentido, coexisten (como centrales o periféricos) en el centro, la periferia y en zonas intermedias. Territorio intermedio se corresponde con lo que Galtung denomina "go-between" y define como una zona de contacto entre el centro y la periferia (p.104); podemos situar ahí un festival que ocupe un lugar periférico con respecto al subcircuito de festivales europeos, pero central en el que constituyen los festivales celebrados periféricamente en América Latina, por ejemplo.

Por su parte, Wallerstein desarrolla un modelo basado en tres elementos (centro, semi-periferia y periferial que va más allá del binomio desarrollo-subdesarrollo / centro-periferia propuesto con anterioridad por las teorías de la dependencia (Wallerstein, 1974, p. 3). El autor define e identifica el papel de la semi-periferia en función del contexto en el que esta es analizada y expone que "las actividades productivas de estos países semi-periféricos están igualmente divididas. En parte, actúan como una zona periférica para los países centrales y, en parte, actúan como un país central para algunas áreas periféricas" (Wallerstein, 1976, pp.462-463).

Wallerstein defiende también la posibilidad que el estatuto de cada territorio cambie, es decir un espacio periférico podría pasar a ser semi-periférico y, más tarde, llegar a ser central. De la misma manera, este proceso podría producirse a la inversa (Wallerstein, 1974). En el contexto de los festivales de cine, esta mutabilidad parece no haber tenido lugar considerando el consenso que existe sobre cómo, desde la década de 1940, los territorios e instituciones cinematográficas centrales serían siempre las mismas y estarían situadas en el eje euro-estadounidense ya mencionado. Del mismo modo, las regiones y espacios asociados a la periferia también continúan estables.

Estos conceptos y relaciones asimétricas planteadas por Galtung y Wallerstein son útiles para analizar el circuito internacional de festivales, así como otras dinámicas propias del campo cinematográfico. Otra propuesta interesante es la desarrollada por Homi $\mathrm{K}$. Bhabha en el marco de los estudios postcoloniales, que identifica en este contexto unos territorios liminales que median entre las regiones colonizadas y colonizadoras, resultando un concepto útil para describir un lugar "intermedio" en el que tiene lugar la transformación cultural [...] el sujeto colonizado puede existir en el espacio liminal entre el discurso colonial y la asunción de una nueva identidad "no-colonial". Pero esta identificación nunca es simplemente el paso de una identidad a otra sino un proceso constante de compromiso, contestación y apropiación (Bhabha, 1994, p.117).

Owen Evans (2007) recupera esta idea del espacio liminal de Bhabha para analizar los festivales de cine, identificando como espacios de mediación que reducen las desigualdades entre unos territorios y cinematografías colonizadoras (centrales) y colonizadas (periféricas). Por nuestra parte, consideramos que, si bien los festivales juegan ese papel de mediadores entre las cinematografías del mundo, no eliminan dichas desigualdades, sino que las mantienen dado que son los festivales de mayor importancia en el contexto internacional - los que constituyen el centro de dicho circuito- los que organizan los cines del mundo en categorías que, muchas veces, responden también a la dicotomía de centro-periferia.

\section{Análisis de festivales de América Latina en el circuito internacional: importancia relativa y subcircuitos}

Nuestra hipótesis es que los grandes festivales se situaron en el centro del circuito internacional en el mismo momento de su creación: Venecia en 1932, Cannes en 1939/46 y Berlín en 1951, y mantienen dicha posición. De acuerdo con esto, serían algunos festivales europeos (y norteamericanos) los que estarían diseñando y construyendo las categorías en las que los cines del mundo se organizan, así como identificando y legitimando nuevas estéticas, olas y cineastas. Al mismo tiempo, otros festivales que despliegan estrategias similares tienen un rol secundario y una menor influencia en esta tarea debido a su menor tamaño, importancia mediática, presupuesto o número de profesionales asistentes, pero también debido a su pertenencia a una región cinematográfica periférica.

Analicemos, en primer lugar, las relaciones entre festivales de América Latina y el circuito internacional considerando las cuatro categorías establecidas por Galtung: centro del centro (C), periferia 
del centro (P), centro de la periferia (c) y periferia de la periferia $(p)^{5}$. Conforme a estas cuatro categorías, la importancia de cada evento sería decreciente cuando perteneciera a cada una de las divisiones. De acuerdo con esta propuesta, podemos hablar de Europa y Norteamérica ${ }^{6}$ como el centro y del resto del mundo como periferia de la cinematografía mundial. Hay festivales que, además de estar en la cima del circuito internacional, son centrales en el contexto europeo (C), caso de Cannes, Venecia y Berlín. Otros se localizan en la periferia de este centro europeo (P), como el festival de San Sebastián, en España. Considerando América Latina una región cinematográfica periférica, identificamos varios festivales que serían centrales en dicho contexto (c): BAFICl y Mar del Plata en Argentina; Guadalajara y Morelia en México; SANFIC en Chile; o $\mathrm{FICCl}$ en Colombia. Por último, otros festivales detentan una posición periférica en este mismo marco ( $p$ ), como es el caso del festival que desde 2002 se celebra en Tandil, en la provincia de Buenos Aires, con el nombre de Tandil Cine.

La Tabla 1 presenta una cartografía de festivales de acuerdo con el esquema de centros y periferias propuesto. Incluye también una clasificación sobre la base de diferentes subcircuitos con el propósito de subrayar la importancia relativa de cada evento según el contexto en el que se analiza.

Tabla 1: Festivales centrales y periféricos

\begin{tabular}{|c|c|c|c|c|}
\hline \multirow{3}{*}{ (SUB)CIRCUITO } & \multirow{3}{*}{ CENTRO } & \multicolumn{3}{|c|}{ PERIFERIAS } \\
\hline & & \multicolumn{2}{|c|}{$\begin{array}{l}\text { SEMIPERIFERIA } \\
\text { (Wallerstein) }\end{array}$} & \multirow{2}{*}{$\begin{array}{c}\begin{array}{c}\text { PERIFERIA } \\
\text { (Wallerstein) }\end{array} \\
\text { PERIFRIA DE } \\
\begin{array}{c}\text { LA PERIFERIA } \\
\text { (Galtung) }\end{array}\end{array}$} \\
\hline & & $\begin{array}{l}\text { PERIFERIA } \\
\text { DEL CENTRO } \\
\text { (Galtung) }\end{array}$ & $\begin{array}{l}\text { CENTRO DE } \\
\text { LA PERIFERIA } \\
\text { (Galtung) }\end{array}$ & \\
\hline $\begin{array}{c}\text { CIRCUITO } \\
\text { INTERNACIONAL }\end{array}$ & $\begin{array}{c}\text { Cannes, Venecia, } \\
\text { Berlín }\end{array}$ & $\begin{array}{l}\text { Rotterdam, } \\
\text { Sundance }\end{array}$ & $\mathrm{BAFICI}$ & $\begin{array}{l}\text { Festival de Cine } \\
\text { de Sucre }\end{array}$ \\
\hline $\begin{array}{l}\text { FESTIVALES } \\
\text { CLASE A } \\
\text { (FIAPF) }\end{array}$ & $\begin{array}{l}\text { Cannes, } \\
\text { Venecia, } \\
\text { Berlín }\end{array}$ & $\begin{array}{l}\text { San Sebastián, } \\
\text { Karlovy Vary, } \\
\text { Locarno, Montreal }\end{array}$ & $\begin{array}{l}\text { Shanghai, Tokio, } \\
\text { Moscú, Mar del } \\
\text { Plata, Goa }\end{array}$ & El Cairo \\
\hline $\begin{array}{c}\text { FESTIVALES } \\
\text { DEDICADOS AL CINE } \\
\text { LATINOAMERICANO }\end{array}$ & $\begin{array}{l}\text { Cinélatino } \\
\text { de Toulouse }\end{array}$ & Huelva & $\begin{array}{l}\text { FESAALP Festival } \\
\text { de Cine Latinoame- } \\
\text { ricano de la Plata }\end{array}$ & $\begin{array}{l}\text { Festival Cero Latitud } \\
\text { (Quito, Ecuador) }\end{array}$ \\
\hline $\begin{array}{l}\text { FESTIVALES INTERNA- } \\
\text { CIONALES CELEBRADOS } \\
\text { EN AMÉRICA LATINA }\end{array}$ & $\begin{array}{c}\text { BAFICI, Mar del } \\
\text { Plata, SANFIC, } \\
\text { Guadalajara, Morelia }\end{array}$ & $\begin{array}{l}\text { Viña del Mar, } \\
\text { Valdivia, } \\
\text { Sao Paulo }\end{array}$ & $\begin{array}{l}\text { Lima, } \\
\text { La Habana }\end{array}$ & $\begin{array}{c}\text { Festival } \\
\text { Internacional FENAVID } \\
\text { (Santa Cruz, Bolivia) }\end{array}$ \\
\hline
\end{tabular}

Fuente: elaboración propia.

La importancia de los festivales es, casi siempre, decreciente en el orden del centro del centro hacia la periferia de la periferia. El énfasis en ese casi se debe al peso que algunos festivales comparten independientemente de su pertenencia a regiones centrales o periféricas. La importancia de los festivales de la periferia del centro $(P)$ y en el centro de la periferia (c) es muy similar. En este sentido, hay una equivalencia entre el festival de San Sebastián en España (periférico en Europa con respecto a Cannes y Berlín) y el BAFICI, en Argentina Icentral en el contexto de los festivales celebrados en América Latina). Desde esta perspectiva, la pe- riferia del centro (P) y el centro de la periferia (c) compartirán un espacio de semi-periferia.

La relativa importancia de cada evento es una de las hipótesis centrales de este trabajo. Podemos considerar, así, que el BAFICI detenta un rol diferente cuando atendemos a su relevancia con respecto al cine y la región latinoamericana, cuando lo analizamos en su relación con el cine hablado en español y cuando lo hacemos en el marco del circuito internacional ${ }^{7}$. En los dos primeros casos, el BAFICl es un evento central a la hora de otorgar visibilidad a películas y cineastas e influir en otros 
festivales que luego seleccionan estos mismos títulos o los futuros trabajos de sus creadores. En el tercer caso, sería periférico con respecto a festivales como Cannes y Berlín. Sergio Wolf (2016), ex director del BAFICI, lo define como un "festival faro" para América Latina dado que, desde su origen, ha influido a otros festivales creados con posterioridad en la región que lo habrían seguido en aspectos organizacionales y de programación (p.82-83). Un estudio sobre los festivales de la región también destaca la influencia del BAFICI sobre otros festivales creados posteriormente (Gutiérrez \& Wagenberg, 2013).

El esquema de Galtung puede también actualizarse considerando las diferentes secciones en que se articula la programación de los festivales. Así, la importancia de la Competición Oficial (C) y la Quincena de Realizadores (P) del festival de Cannes, podrían oponerse a la Competición Internacional del BAFICI (c) y a Panorama (p), organizada por el mismo festival. Lo mismo ocurre al atender a la Competición Oficial (C) y Forum (P) de la Berlinale y la Selección Oficial (c) y la sección Mejor música en una película chilena (p) del Festival de Viña del Mar, en Chile.

El escenario anterior se repite en otros mercados, iniciativas y actividades organizadas por festivales de todo el mundo. También es el caso de los fondos de financiación y talleres puestos en marcha desde la década de 1990 y que constituirán, entre todos, un subcircuito específico. El esquema de centros y periferias propuesto es adecuado para estudiar los diálogos y sistemas de influencias que tienen lugar en este subcircuito específico. Podríamos hablar aquí de la importancia relativa que para diferentes cinematografías tienen el Fondo Hubert Bals de Rotterdam, que situamos en el centro del centro (C), el papel secundario del fondo organizado por el Festival de Amiens (P), los talleres organizados en el marco del Buenos Aires Lab del $\mathrm{BAFICI}$ (c) o, por último, las sesiones de proyectos en desarrollo organizadas por el Festival Asterisco de Buenos Aires ( $p$ ).

De acuerdo con lo expuesto, podremos analizar la importancia y visibilidad que una película recibe al ser programada en una sección particular de un festival concreto. Así, si participa en la Competición Oficial de Cannes daría como resultado la figura $\mathrm{C}>\mathrm{C}$ : una sección central en un festival central del circuito internacional. Siguiendo este mismo ejemplo, una película programada en una sección retrospectiva en el BAFICI presentaría la figura $c>p$ : una sección periférica de un festival central en su contexto regional periférico si tomamos como referencia el circuito internacional. Si, por el contrario, tomamos como marco del análisis el subcircuito de festivales celebrados en América Latina o el de festivales dedicados al cine en español, el último caso resultaría en la figura $C>P$, dado que el BAFICl está a la cabeza de ambos subcircuitos y que una sección retrospectiva sería siempre periférica en la programación de cualquier festival. Por ello, son distintos los festivales y las posiciones centrales y periféricas en función de los marcos del análisis y los subcircuitos considerados.

Estos esquemas y relaciones desiguales condicionan elementos clave en el contexto cinematográfico internacional en conjunto con las políticas estatales de fomento y apoyo a la internacionalización y las estrategias de distribución diseñadas para cada película. En el primer caso, son muchos los organismos que cuentan con una línea específica de acción destinada a la participación de películas nacionales (y sus responsables) en mercados y festivales internacionales. A la hora de distribuir el presupuesto anual entre las películas candidatas, estos organismos cuentan con tablas en las que el monto de la ayuda es mayor o menor en función del festival y la sección para la que ha sido seleccionada la película. En la mayoría de los casos, los organismos elaboran sus propias categorías en función de la importancia que otorgan a cada festival y sección, aunque muchas veces están inspiradas en las que establece la Federación Internacional de Asociaciones de Productores de Cine (FIAPF). Es el caso de los estímulos de México, Brasil, Colombia, Chile y Uruguay; también de Argentina, donde la cuantía para participar en alguno de los festivales considerados clase A (Berlín, Cannes, Venecia y San Sebastián) varía si la película se programa en una Competencia oficial (hasta 50.000 USD), una Sección Competitiva Paralela (hasta 25.000 USD) y una Sección No Competitiva (hasta 8.000 USD) ${ }^{8}$.

Del mismo modo, la importancia que la industria otorga a cada festival condiciona las estrategias de distribución, aunque aquí nos refiramos únicamente a la distribución internacional en festivales. Podemos señalar trabajos anteriores que han analizado esto mismo en torno a cines nacionales y regionales y su presencia en el circuito internacional. En una primera aproximación al problema, en 1994. 
Manthia Diawara señalaba que el interés de festivales europeos y norteamericanos por el mejor cine africano hacía que los realizadores dejaran de mirar al Festival Panafricano de Ouagadougou (FESPACO) para estrenar sus películas (Diawara, 1994). Una dinámica que limita la influencia del FESPACO a la hora de incorporar nuevos relatos y cineastas africanos al circuito internacional, más aún si tenemos en cuenta que prefieren ser programados con anterioridad en festivales euro-norteamericanos porque lo consideran más importante para la distribución de sus películas. Esto mismo se repite en otras cinematografías. En su estudio sobre la internacionalización del cine chileno reciente, María Paz Peirano (2018), aunando las dos líneas aquí señaladas, expone que la estrategia de los últimos años del cine chileno a través de la agencia CineChileno ha sido llevar las películas y cineastas al circuito internacional y menciona, como ejemplo, la filmografía de Sebastián Lelio.

\section{El problema de los guetos de programación}

Festivales y secciones paralelas a veces son considerados guetos de programación: espacios que serían contraproducentes para las películas porque actuarían en contra de su visibilidad y valorización (Diawara, 1994). Los festivales situados en el centro del sistema influyen en eventos periféricos y estas sinergias también ocurren entre los festivales del centro y entre los ubicados en la periferia en la industria audiovisual. Tengamos en cuenta los casos de Cannes (como festival C), el BAFICI (c), Cinélatino de Toulouse (P) y Tandil Cine (p): la influencia de Cannes sobre BAFICl y Cinélatino, estos dos festivales podrían influirse entre ellos lal considerar c y $\mathrm{P}$ como equivalentes) y los tres podrían inspirar a Tandil Cine. Sin embargo, la influencia podría producirse también en un sentido inverso, desde las periferias hacia el centro: una película estrenada en Tandil Cine (p), que es programada más tarde en el BAFICI (c) y Cinélatino (P), en alguna de sus secciones diferentes de la Competición Oficial, podría llegar a programarse en alguna edición posterior del festival de Cannes (C), quizá como parte de una retrospectiva.

A partir de esta influencia a la inversa podemos revisar la idea de guetos de programación en relación con el cine africano (Diawara, 1994). Es realmente difícil que una película estrenada en Tandil Cine (p) sea más tarde programada en Cannes (C); sin embargo, es posible. Como señala Diawara, el hecho que los productores y creadores estén interesados en enviar sus obras a los festivales más importantes del circuito internacional está en el origen de la concepción de ciertos festivales y secciones como menos relevantes. Están interesados en ubicar sus películas en los festivales más importantes de un subcircuito determinado, así como en la sección más relevante de cada evento (en detrimento de secciones paralelas o no competitivas). Entre las secciones competitivas, hay algunas de mayor interés porque ofrecen premios más importantes y cuantiosos (Czach, 2004). Las secciones principales cuentan con un lugar privilegiado tanto en los catálogos como en los espacios físicos del festival, donde los horarios y las pantallas suelen ser también los mejores. Son éstos algunos de los motivos, junto con la atención mediática que despiertan, por los que creadores y productores prefieren las secciones oficiales y competitivas de cada festival en detrimento de otros espacios paralelos o retrospectivos. Sin embargo, y como hemos visto, esta tendencia no impide que la influencia se produjera desde festivales a priori menos relevantes en el circuito internacional hacia otros de mayor rango y, por este motivo, convendría también relativizar el carácter de guetos de programación asociados a algunos espacios.

\section{La importancia del diálogo centro-periferia en el discurso cinematográfico}

Evans (2007) define los festivales como espacios de mediación entre diferentes regiones cinematográficas. Aunque coincidimos en esta idea, estimamos que los festivales de cine también mantienen estas diferencias entre las regiones centrales y periféricas. Festivales situados en el centro del circuito internacional con frecuencia influyen en las tendencias cinematográficas y las regulaciones del resto de los eventos. Compartimos también con Evans su consideración sobre los festivales como espacios liminales y lugares de "compromiso, respuesta y apropiación" (Ashcroft, Griffiths \& Tiffin, 2007, pp. 117-118). A través de estos procesos, los festivales crean determinadas categorías cinema- 
tográficas para organizar sus programas y secciones basados en criterios tales como el territorio de origen de las películas, su formato o sus temáticas. La clave en este proceso está en que todos los festivales del circuito internacional tengan la capacidad de proponer sus propias categorías de programación y sus cánones, aunque no todos ellos tengan el mismo poder en esta tarea ni la influencia suficiente para afectar al circuito de festivales en su conjunto (o en un amplio espectro).

Siguiendo estos argumentos, podemos determinar que el papel que cada festival juega en la creación de cánones y categorías cinematográficas en el contexto general depende de su pertenencia al centro o la periferia del circuito. De esta manera, las instituciones europeas y estadounidenses y los festivales del centro del circuito internacional (Cannes, Venecia y Berlín) tendrían mayor capacidad y poder para construir dichas categorías e identificar tendencias, así como para consolidarlas y legitimarlas.

A partir de la coproducción hispano-peruana Madeinusa (Llosa, 2006) podemos identificar las fuerzas desiguales que aparecen en los procesos señalados. Cada vez que un festival programa una película, lo hace bajo una categoría determinada que tiene que ver con el título de la sección o con la propia línea del evento, así, con cada selección, se irían añadiendo a las películas "etiquetas" que las acompañan cuando son programadas en otros festivales y en otras pantallas. Si bien este "etiquetado" tiene lugar en la crítica, los museos, las galerías, las instituciones culturales y los materiales y campañas promocionales de las películas, en este caso solo atenderemos al espacio del festival. Así, después de su estreno en el Festival de Rotterdam en 2006, Madeinusa ha sido programa en secciones como "Territorio latinoamericano" en el Festival de Málaga (2006, España), “Femmes du Cinéma” (Mujeres de cine) en el festival Cinélatino de Toulouse (2014, Francia) y en NATIVe- A Journey into Indigenous Cinema en la edición de 2013 de la Berlinale. En el primer caso, se programó como una película latinoamericana laun siendo una coproducción con España); en el segundo, como una película dirigida por una mujer; y, en el tercero, como una película indígena launque su inclusión en esta categoría resulte controvertida). En los tres casos, las diferentes etiquetas fueron añadidas progresivamente a Madeinusa y lo son, frecuentemente, cuando se menciona y programa la película en diferentes contextos. Este proceso taxonómico sería, al mismo tiempo, una operación política que nos obliga a prestar atención al modo en el que las relaciones desiguales que existen entre las instituciones cinematográficas deciden, construyen, transfieren y legitiman categorías de interpretación de diferente naturaleza.

\section{Conclusión}

En este trabajo hemos querido atender a lo problemático de las categorías de centro y periferia en el marco de los estudios cinematográficos y a su operatividad al analizar la influencia y peso que cada festival de cine tiene en el contexto internacional a pesar de haber sido señaladas, igual que las categorías vinculadas a lo nacional, como obsoletas para abordar una realidad y una industria globales. Nuestro objetivo, una vez que la oposición centroperiferia se mantiene en los discursos sobre cine contemporáneo, es subrayar los diálogos desiguales y las fricciones que este binomio implica y la necesidad de abordar cinematografías, instituciones y agentes pertenecientes a cada categoría siempre en base a sus relaciones con la otra.

Por esto mismo, y superando la polarización más radical que implican los términos centro-periferia, hemos querido destacar los espacios intermedios propuestos por Immanuel Wallerstein y Johan Galtung así como algunas de sus ideas sobre la dirección de las influencias en este tipo de relaciones asimétricas, la existencia de centros y periferias simultáneos y el posible cambio en el estatuto de cada una de las regiones consideradas centrales y periféricas. Todos estos fenómenos son visibles e identificables en el campo de los festivales de cine y válidos para el análisis de dinámicas y problemas que les son propios.

Por último, cabe señalar que el modelo propuesto y los marcos teóricos considerados en este trabajo, no exentos de algunos problemas mencionados a lo largo del texto, serían igualmente válidos para el estudio de otras instituciones y circuitos cinematográficos y culturales, aunque aquí hayamos puesto el foco en los festivales y el cine de América Latina. 


\section{Notas}

1. Los primeros festivales de cine fueron creados precisamente en el marco de festivales culturales.

2. La idea de los festivales de cine como un circuito de distribución inicial puede ser revisada y actualizada en la medida en que las secciones paralelas y retrospectivas permiten a los festivales recuperar películas estrenadas en temporadas anteriores.

3. Osvaldo Sunkel incluye ambos conceptos en una larga lista de términos opuestos y relativos al mundo desarrollado y subdesarrollado que “[...] interactúan y se condicionan entre ellos; y cuya expresión geográfica se concreta en dos grandes polarizaciones. Por un lado, la polarización del mundo en países industriales, avanzados, desarrollados y centrales y países subdesarrollados, atrasados, pobres, periféricos y dependientes [...]" (Sunkel, 1971, p.9).

4. Nombraremos: C: centro del centro; P: periferia del centro; c: centro de la periferia; p: periferia de la periferia.
5. Para los ejemplos y casos citados consideramos el impacto que los festivales han tenido en la emergencia y consolidación de los llamados novísimos cines latinos, la bibliografía especializada, el estudio de datos empíricos y los resultados de una investigación anterior (Campos, 2016a).

6. Junto con la importancia de la industria estadounidense, consideramos también la importancia que tiene un festival como el de Toronto.

7. Autores como Bill Nichols han señalado la posibilidad de que un mismo festival internacional detente una posición marginal en términos geográficos, pero central en términos culturales para una cinematografía concreta (Nichols, 1994, p.74).

8. Datos de la convocatoria del Instituto Nacional de Cine y Artes Audiovisuales de 2012 (Campos, 2016a, pp.238-239).

9. Entonces, Rencontres Cinémas d’Amérique Latine.

\section{Referencias}

Appadurai, A. (1996 [2001]). Modernidad desbordada. Dimensiones culturales de la globalización. Buenos Aires: Fondo de Cultura Económica.

Ashcroft, B., Griffiths, G. \& Tiffin, H. (Eds.) (2007). Post-colonial Studies. The Key concepts. Nueva York: Routledge.

Bhabha, H. K. (1994 [1989]). The Location of Culture. Londres-Nueva York: Routledge.

Campos, M. (2016a). Construcción y legitimación de los cines (trans)nacionales en el circuito internacional de festivales. El caso de América Latina [tesis doctoral no publicada]. Universidad Carlos III de Madrid. https://e-archivo.uc3m.es/handle/10016/23801

Campos, M. (2016b). Presentación. Alberto Elena y los cines periféricos. Secuencias. Revista de historia del cine, (43-44), 7-19. https://revistas.uam.es/secuencias/article/ view/7294/8236

Campos, M. (2018). Lo (trans)nacional como eje del circuito de festivales de cine. Una aproximación histórica al diálogo Europa-América Latina. Imagofagia. Revista de la Asociación Argentina de Estudios de Cine y Audiovisual, (17), 11-40. http://www.asaeca.org/ imagofagia/index.php/imagofagia/article/view/1441/1315

Crofts, S. (1999). "Concepts of National Cinema". En J. Hill \& P. Church Gibson (Eds.), The Oxford Guide to Film Studies, (pp. 385-394). Oxford: Oxford University Press. 
Crofts, S. (2006 [1993]). Reconceptualising National Cinema/s. En V. Vitali \& P. Willemen (Eds.), Theorising National Cinema, (págs. 44-58). Londres: British Film Institute.

Czach, L. (2004). Film Festivals, Programming, and the Building of a National Cinema. The Moving Image, 4(1), 56-88.

De Valck, M. (2007). Film Festivals: From European Geopolitics to Global Cinephilia. Amsterdam: Amsterdam University Press.

De Valck, M. (2014). Supporting art cinema at a time of commercialization. Principles and practices, the case of the international Film Festival Rotterdam. Poetics, 42, 40-59.

Diawara, M. (1994). On Tracking World Cinema: African Cinema at Film Festivals. Public Culture, 2(6), 385-396.

Elena, A. (1999). Los cines periféricos. África, Oriente Medio, India. Barcelona: Paidós.

Evans, O. (2007). Border Exchanges: The Role of the European Film Festival. Journal of Contemporary European Studies, 15(1), 23-33.

Falicov, T. (2017). "The Interlocking Dynamics of Domestic and International Film Festivals: The Case of Latin American and Caribbean Cinema". En M. D'Lugo \& L. Podalsky (Eds.), Routledge Companion to Latin American Cinemas (pp. 266-277). Londres: Routledge.

Fléchet, A., Goetschel, P., Hidiroglou, P., Jacotot, S., Moine, C. \& Verlaine, J. (Dirs.) (2013). Une Histoire des festivals. XXe-XXIe Siècle. París: Publications de la Sorbonne.

Galtung, J. (1971). A Structural Theory of Imperialism. Journal of Peace Research, 8(2), 81-117. http://bev.berkeley.edu/ipe/readings/galtung.pdf

Gutiérrez, C. \& Wagenberg, M. (2013). Meeting Points: A Survey of Film Festivals in Latin America. Transnational Cinemas, 4(2), 295-305.

Harbord, J. (2002). Film Cultures. Londres: SAGE Publications.

Iordanova, D. (2009). "The Film Festival Circuit". En D. Iordanova \& R. Rhyne (Eds.), Film Festival Yearbook 1: The Festival Circuit, (pp. 23-39). St. Andrews: St. Andrews Film Studies.

Latour, B. (2008 [2005]). Reensamblar lo social: una introducción a la teoría del actor-red. Buenos Aires: Manantial.

Nichols, B. (1994). Global Image Consumption in the Age of Late Capitalism. East-West, 8(1), 68-85.

Peirano, M. P. (2018). Festivales de cine y procesos de internacionalización del cine chileno reciente. Cuadernos.info, (43), 57-69. https://doi.org/10.7764/cdi.43.1485

Ross, M. (2011). The film festival as producer: Latin American Films and Rotterdam's Hubert Bals Fund. Screen, 52(2), 261-267.

Stringer, J. (2001). "Global Cities and the International Film Festival Economy". En M. Shiel \& T. Fitzmaurice (Eds.), Cinema and the City. Film and Urban Societies in a Global Context (pp. 134-144). Oxford: Blackwell. 
Stringer, J. (2016). "Film festivals in Asia: notes on history, geography, and power from a distance". En M. de Valck, B. Kredell, y S. Loist (Eds.) Film Festivals. History, Theory, Method, Practice (pp. 34-48), Londres-Nueva York: Routledge.

Sunkel, O. (1971). Capitalismo transnacional y desintegración nacional. Estudios Internacionales, (16), 3-61. http://www.jstor.org/stable/41390713

Taillibert, C. \& Wäfler, J. (2016). Groundwork for a (pre)history of film festivals. New Review of Film and Television Studies, 14(1), 5-27.

Vallejo, A. \& Peirano, M. P. (Eds.) (2017). Film Festivals and Anthropology. NewCastle upon Tyne: Cambridge Scholars Publishing.

Wallerstein, I. (1974). Dependence in an Interdependent World: The Limited Possibilities of Transformation within the Capitalist World Economy. African Studies Review, 17(1), $1-26$.

Wallerstein, I. (1976). Semi-Peripheral Countries and the Contemporary World Crisis. Theory and Society, 3(4), 431-483.

Wolf, S. (2016). "El BAFICl como ventana al mundo del cine argentino". En R. Lefere \& N. Lie (Eds.), Nuevas perspectivas sobre la transnacionalidad del cine hispánico (pp. 82-85). Leiden-Boston: Brill / Rodopi.

\section{- Sobre la autora:}

Minerva Campos Rabadán es investigadora postdoctoral Juan de la Cierva-Formación en la Universidad Autónoma de Madrid. Doctora en Comunicación Audiovisual por la Universidad Carlos III de Madrid, es miembro de los grupos de investigación DeVisiones (UAM) y TECMERIN (UC3M).

\section{- ¿Cómo citar?}

Campos-Rabadán, M. (2020). Tensiones en el circuito cinematográfico internacional: modelo para el estudio de los festivales latinoamericanos. Comunicación y Medios, (42), 72-84. https://doi.org/10.5354/0719-1529.2019.57258 